\title{
Early lung cancer detection based on registered perfusion MRI
}

\author{
HENG HUANG $^{1}$, LI SHEN $^{3}$, JAMES FORD $^{1}$, LING GAO $^{2}$ and JUSTIN PEARLMAN ${ }^{1,2}$ \\ ${ }^{1}$ DEVLAB, Department of Computer Science, Dartmouth College, Hanover, NH 03755; ${ }^{2}$ Departments of Medicine and \\ Radiology, Division of Cardiology, Dartmouth Medical School, Lebanon, NH 03756; ${ }^{3}$ Computer and Information Science \\ Department, University of Massachusetts Dartmouth, MA 02747, USA
}

Received July 7, 2005; Accepted September 29, 2005

\begin{abstract}
Lung cancer remains the most common fatal malignancy in both men and women in the United States and elsewhere around the world among people who have been exposed passively or actively to tobacco smoke. Technological advances have produced imaging modalities that are proving to be useful for the early detection of lung cancer. In the usual modes, computed tomography (CT) and magnetic resonance imaging (MRI) can identify suspicious lesions, but further work is needed to detect cancer in its early stage. Tumor angiogenesis assessed by perfusion-sensitive MRI is a promising method for early and accurate identification of lung cancer that avoids patient stress and the potential progression to a less treatable stage inherent in serial imaging. However, compensating for the respiratory and anatomical structure motion is a challenge. In order to use perfusion MRI in a signal imaging session to define metabolic and vascular characteristics of tumors, we present a novel affine registration method for perfusion MRI that can register points of interest by tracking image intensity changes around the target point. The registration results are used to generate accurate time intensity curves (TIC) of different regions of interest (ROI). The patterns of different TICs are mapped on the cancer and other structures. The method is computationally efficient and performs well in our perfusion MRI sequence analysis.
\end{abstract}

\section{Introduction}

Lung cancer is the most common carcinoma in both men and women and the one that causes the most deaths; lung cancer deaths annually exceed cancer deaths due to breast, prostate and colon cancer combined. To combat this often

Correspondence to: Dr Heng Huang, DEVLAB, Department of Computer Science, Dartmouth College, Hanover, NH 03755, USA

E-mail: hh@cs.dartmouth.edu

Key words: perfusion magnetic resonance imaging, lung cancer detection, medical image registration, time intensity curve lethal cancer, it is important to detect lung cancer in its early stages, when it is more curable and survival is more likely.

Developments in MR technology during the past decade have made it possible to acquire new information on dynamic processes in the human body. Perfusion magnetic resonance imaging (pMRI) is an imaging technique used for measuring blood flow through the organs, tissues, and vessels (1). MRI of pulmonary perfusion has become feasible by using dynamic contrast-enhanced imaging with an inversion recovery (IR) Turbo-Fast Low-Angle SHot (FLASH) sequence (2). When the scanner continuously acquires data, the paramagnetic contrast agent is injected and perfuses through the lung of the patient. This technique has evolved into an attractive alternative to other non-invasive methods that involve radiation exposure $(3,4)$. Perfusion MRI offers a method for improving early detection of the highly fatal lung cancer.

Perfusion MR image sequence often suffers from the motion induced by breathing during image acquisition. To ensure that corresponding anatomical structures in different time frames can be properly compared, registration of the pMRI sequence is necessary. For example, the tumor marked by a circle in Fig. 1 is moving up as the patient breathes. This fact directly limits the accurate monitoring of tumor evolution by pMRI sequence.

Image registration is the process of aligning two or more images of the same scene taken at different times or from different viewpoints. Many registration algorithms have been proposed for medical image analysis in previous studies. In these algorithms, landmarks (5), contours (6) and surfaces (7) are used to manually, semi-automatically or automatically define correspondences between two or more images. Perfusion MRI sequence is made into gray color images. The anatomical information is provided by gray values rather than shapes and structures. Thus, correlation-like registration methods are preferably applied to such medical image (8). In previous approaches, people use windows with a predefined size or even entire images for the correspondence estimation $(9,10)$.

In this study, we present a new method for image registration based on comparing the gray value sequence around the point of interest (landmarked by people) to the gray value sequence of points in the remaining image frames. The cross correlation method is used to measure the distance between each gray value sequence pair. This method produces the registered time-intensity curves (TIC; 

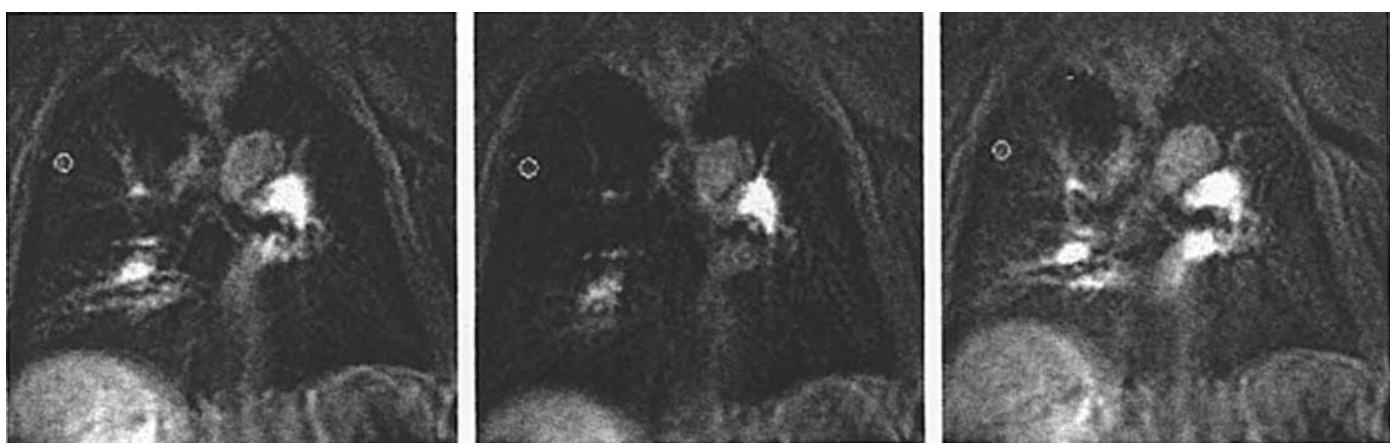

Figure 1. Lung perfusion MRI. The tumor region is marked by a circle that is moving down from left image to mid-image and moving up from mid-image to right image as the patient breathes.

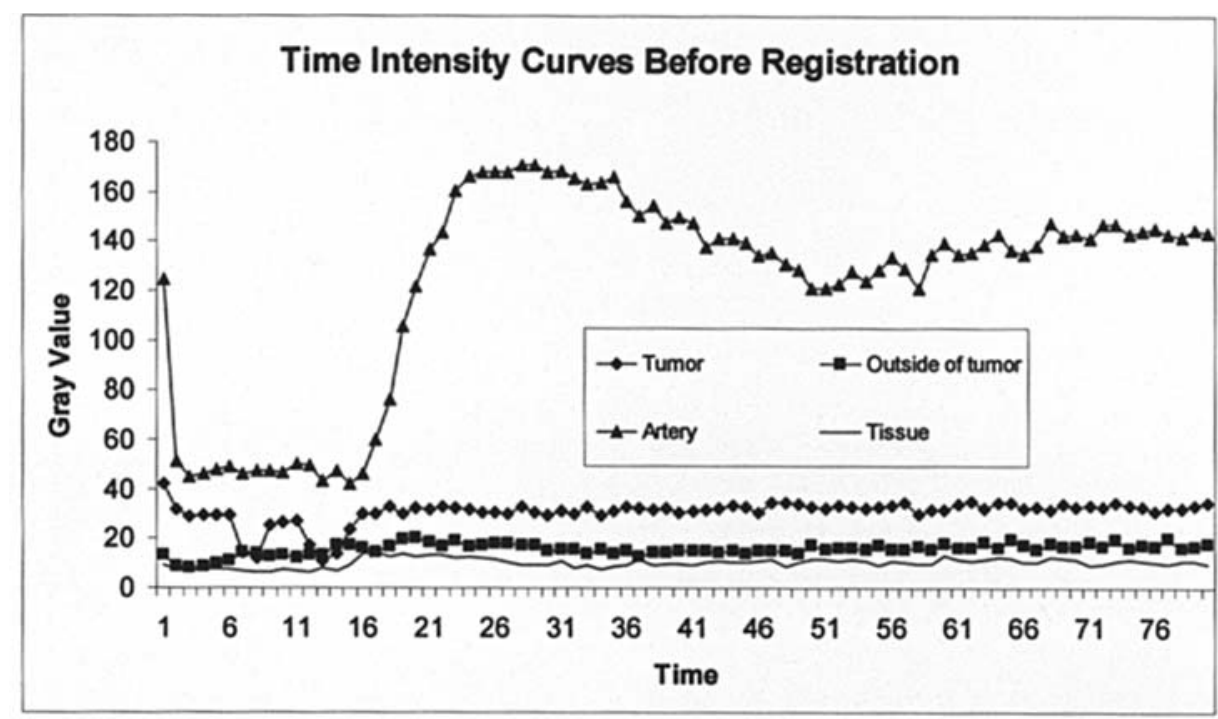

Figure 2. Time intensity curves before registration. The curves are generated by selecting the target in the first image and sampling the following points on the remainder of the image sequence with the same coordinates.

i.e. curves in Fig. 2) of the region of interest (ROI) in the pMRI sequence. The results show that TIC analysis after registration can distinguish the tumor from anatomical structures.

\section{Materials and methods}

MRI protocol. The data comprise 800 lung perfusion, shortaxis, MR images obtained from ten freely breathing patients. For each subject, 80 sequential images were acquired after a bolus of gadopentetate dimeglumine, commercially known as Magnevist (Berlex Laboratories, Wayne, NJ), as the contrast agent using an inversion recovery (IR) Turbo-FLASH MR sequence on a $1.5 \mathrm{~T}$ Siemens Vision prototype (Siemens, Erlangen, Germany). The pMRI sequences of lung images are produced in the DICOM format with a $256 \times 256$ pixel size, i.e. images in Fig. 1.

Data analysis. Different approaches have been proposed for perfusion measures from contrast-enhanced MRI. TIC is a graph of the time course of signal intensity changes due to the passage of the blood-borne contrast agent through the lung. Thus, it can be used as a reliable index of quantitative assessment of lung pMRI data. By comparing signal intensity (SI) from a specific ROI to that from normal tissue, or by computing a map showing contrast, blood arrival time, blood flow or other measures derived from the TICs, we can determine regional impairment of the blood supply. Based on these results, we can separate the tumor from other tissues and detect lung cancer in its early stage.

Because breathing causes body motion, the functional information represented by these TICs is disturbed. For example, in Fig. 2, the diamond curve dips in image frames (timing phase) 6-7 and 12-13. The fundamental problem is the tumor moves in the images, and gray values from these four frames are incorrect for the selected tumor. Therefore, registration must be performed for the ROI before the TICs are computed from pMRI for different tissues.

Registration method. In order to assess the corresponding anatomical structures in different time frames, we propose a novel registration method. The pMRI is stored as an intensity image represented by a data matrix. There are eight neighboring points for each point (except for points on the border of image), which are denoted as level 1 neighbor points. Outside these eight points, there are sixteen others considered to be level 2 neighbor points. For the $i$-th level neighbor points, we know the number of these points is $8 x i$. Given a point $s$ as the ROI of image, we use level intensity value $f_{\mathrm{i}}$ to hold all intensity values of the $i$-th level neighbor points of $s$ as 
$f_{\mathrm{i}}=\{x 1 \ldots x 81\}$ where $x_{\mathrm{j}}$ is the intensity value of one $i$-th level neighbor point. Meanwhile, we define the position value $f$ of point $s$ from its neighbor point intensity values as $f=\left\{f_{1} \ldots f_{n}\right\}$, where $n$ is decided by the number people of which 6 were used during our experiments.

We treat the position value $f$ as a value sequence. For each level intensity value $f_{\mathrm{i}}$, the starting point is the leftmost top point and the order of the other points goes along $i$-th level points anti-clockwise. In order to compare the position value between different lung pMRI, we use a distance function to represent the similarities between them. It is important to pick an appropriate distance function. In this case, we observe that the Euclidean distance is not sensible enough and a better choice is to use the cross correlation to measure the distance between every sequence pair. Formally, given two intensity value sequences $\vec{x}=\left\{x_{1}, \cdots, x_{n}\right\}$ and $\vec{y}=\left\{y_{1}, \cdots, y_{n}\right\}$ we employ the following formula to measure the distance or similarity between them:

$$
d(\vec{x}, \vec{y})=\vec{x} \circ \vec{y}=\sum_{m=1}^{n} x(m) y(m+t),
$$

where ' $\mathrm{o}$ ' is the correlation operator, and $\mathrm{t}=0 \ldots \mathrm{n}-1$. The time shift $t$ is called the correlation lag, and $x(m) y(m+t)$ is called a lagged product (11). If $t=t_{0}$ satisfies

$$
d(\vec{x}, \vec{y})\left(t_{0}\right)=\max (d(\vec{x}, \vec{y})(t)) \text { for } t \in[0, n-1],
$$

then the intensity value sequence $x$ shifts to get the maximum correlation with the intensity value sequence $y$. We define this shift value $t=t_{0}$ as the distance between these two intensity value sequences.

The cross correlation distance can be computed efficiently using the Fast Fourier Transform (FFT). According to the correlation theorem, $d(\vec{x}, \vec{y})=\vec{x} \circ \vec{y}=\operatorname{InverseDFT}(\overrightarrow{D F T(\vec{x})} D F T(\vec{y}))$, where $\operatorname{DFT}(\vec{x})$ is the complex conjugate of $\operatorname{DFT}(\vec{x}), \operatorname{DFT}(\vec{y})$ the Discrete Fourier Transform of $\vec{y}$, and InverseDFT $(\overrightarrow{D F T(\vec{x})} D F T(\vec{y})$ the Inverse Discrete Fourier Transform of $\overrightarrow{\operatorname{DFT}(\vec{x})} \operatorname{DFT}(\vec{y})$ (11).

This allows us to achieve the computational benefit. The time complexity is reduced to $O(n \log n)$ rather than the $O\left(n^{2}\right)$ time complexity of the brute force approach of shifting and comparing the intensity value sequences. When we perform registration for the points of interest in a large sized pMRI, the computational time will be significantly reduced to achieve fast registration.

During the registration step, the ROI point $s$ is selected on the first image of the pMRI sequence. Corresponding points in the other images for point $s$ need to be identified. If the corresponding points are known before image $k$ in the sequence, the area around this coordinate in the next image $k+1$ will be examined to find the point with the minimum position value distance between it and the point on image $k$. Since breathing only causes the chamber and other tissues to shift a maximum of 10 pixels up or down during the pMRI, the $10 \times 10$ grid around the target point of each image frame is searched according to the coordinate obtained from the preceding image.

\section{Results}

In pMRI, changes in image intensity during the bolus passage reflect the kinematics of the contrast agent, the behavior of which is tied to functional activity. The dynamics of lung pMRI signal intensity changes can be used to detect nodules, and potentially identify tumor candidates early.

To examine the effect of TIC analysis on lung tumor detection using the pMRI sequence, the TIC of the tumor are compared to that of other anatomical structures (artery, tissue around tumor, etc.). Their TICs before registration (Fig. 2) are generated by selecting the starting point in the first image and sampling the following points on the remainder of the image sequence with the same coordinates. In Fig. 2, the triangle curve represents the movement of the contrast agent in the artery. A transportation delay in the arrival of the contrast agent creates a trough in this time intensity curve. The diamond curve is the TIC of the tumor as measured from the center point of the circle in Fig. 1 through the pMRI sequence. Because many capillary vessels are generated around an active tumor, the diamond curve has a higher value than the tissue curve (line curve). The tissue surrounding a tumor has a mid-level (between the tumor and normal tissue) gray value shown in the square curve. Since breathing causes body motion, the functional information represented by these TICs is inaccurate. Because the tumor is very small in the early stage, the motion caused by breathing is enough to generate incorrect signal intensity values in the TIC. Therefore, in Fig. 2 the diamond curve (tumor TIC) drops in image frames 6-7 and 12-13. For the other anatomical structures, such motion does not signficantly affect their TIC since they consist of large areas.

The registration method described above was applied to the point of interest in the pMRI sequence. By using the registered pMRI images, time intensive curves that represent the correct intensity values of different types of anatomical structures were obtained. Fig. 3 shows the registration results of the pMRI image sequence. In the first frame, one point is selected as the point of interest. The registered points are shown in image frames 2 and 3 for the selected point in frame 1 . They represent the correct positions of the point of interest in the pMRI sequence and do not overlap points (shown as a 'selected point' in the other two frames) having the same coordinate in frame 1.

Using the TICs of registered points, we can find the curve pattern and separate the tumor from other anatomical structures. In Fig. 4, the TIC of the artery (triangle curve) keeps the same trend shown in Fig. 2 because the artery has a large region, and the body motion caused by breathing affects its TIC very little. The same condition appears in the TICs of different tissues (square curve and line curve). The TIC of the tumor (diamond curve) clearly deviates from the TICs of tissues, and there is no overlap.

For the pMRI sequence, several regions were selected as ROIs in the first image. For each point of these regions, registration was performed for every point on the other image frames during the pMRI sequence. The TICs of all points were drawn after registration. TICs of the artery and tumor are easily found with higher intensity values that explicitly separate them from other tissues. For these two kinds TICs, the TIC of the artery can easily be recognized by its intensity trough pattern. Therefore, the TIC analysis after registration can help find a small tumor in the pMRI sequence, as long as the tumor is a certain size (approximately $>2 \mathrm{~mm}$ ) on the image. 

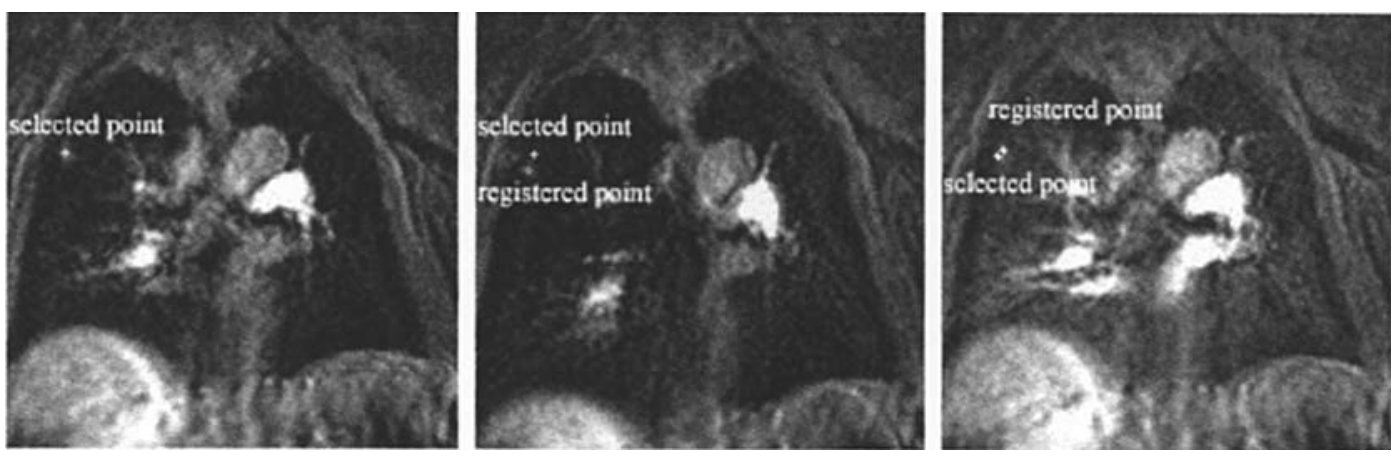

Figure 3. The registration results of pMRI image sequence. In the left image, one point is marked as the interest point. In the other two images, the selected points have the same coordinate of point in left image (without considering the breathing motion) and the registered points are the correct positions of the selected point in left image.

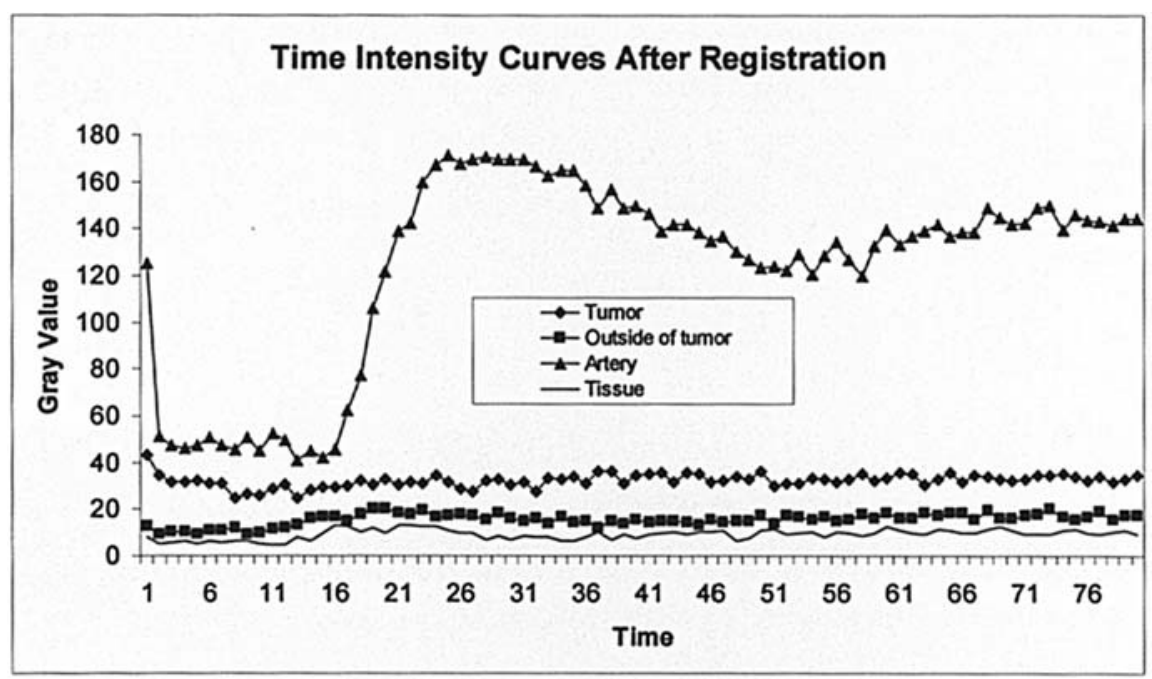

Figure 4 . The time intensity curve analysis after registration.

\section{Discussion}

In this study, we evaluated the performances of TICs of points of interest for tumor detection based on contrast-enhanced MRI studies. Because patients breathe during the MRI scan process, the usefulness of TIC analysis is reduced by the motion of anatomical structures. We used a new registration method to collect corresponding points in the pMRI sequence for the selected point in the first image. As a result, a more accurate TIC is generated for the point of interest. Since an active tumor causes the generation of capillary vessels in its surrounding area, the TIC of a tumor can be distinguished from the TICs of other tissues. The registration results enhance the efficacy and importance of early lung cancer detection by pMRI sequence.

Since the tumor is very small during early stages of lung cancer (1-3 mm), noise points on the pMRI may confuse cancer detection. Future studies will be conducted on the more robust registration method, which should work without concern for the quality of MRI, and will be combined with the current TIC analysis method to improve the accuracy and practicability of early lung cancer detection.

\section{Acknowledgements}

This work is supported in part by FAMRI and the Doris Duke Foundation.

\section{References}

1. Fink C, Puderbach M, Bock M, et al: Regional lung perfusion: assessment with partially parallel three-dimensional MR imaging. Radiology 231: 175-184, 2004.

2. Berthezene Y, Croisille P, Wiart M, Howarth N, Houzard C, Faure O, Douek P, Amiel M and Revel D: Prospective comparison of MR lung perfusion and lung scintigraphy. J Magn Reson Imaging 9: 61-68, 1999.

3. Nikolaou K, Schoenberg S, Brix G, et al: Quantification of pulmonary blood flow and volume in healthy volunteers by dynamic contrast-enhanced magnetic resonance imaging using a parallel imaging technique. Invest Radiol 39: 537-545, 2004.

4. Van Beek E, Wild J, Fink C, et al: MRI for the diagnosis of pulmonary embolism. J Magn Reson Med 18: 627, 2003.

5. Alhichri HS and Kamel M: Virtual circles: a new set of features for fast image registration. Pattern Recog Lett 24: 1181-1190, 2003.

6. Andersen PR and Nielsen M: Non-rigid registration by geometryconstrained diffusion. Med Image Anal 5: 81-88, 2001.

7. Audette MA, Ferrie FP and Peters TM: An algorithmic overview of surface registration techniques for medical imaging. Med Image Anal 4: 201-217, 2000.

8. Zitová B and Flusser J: Image registration methods: a survey. Image Vision Comput 21: 977-1000, 2003.

9. Althof RJ, Wind MGJ and Dobbins JT: A rapid and automatic image registration algorithm with subpixel accuracy. IEEE Trans Med Imaging 16: 308-316, 1997.

10. Maintz JBA and Viergever MA: A survey of medical image registration. Med Image Anal 2: 1-36, 1998.

11. Smith JO: Mathematics of the Discrete Fourier Transform (DFT), 2003. W3K Publishing, http://www.w3k.org/books/. 